\title{
Re: How a Donor Nephrectomy Population Can Help Give Perspective to the Effects of Renal Parenchymal Preservation During Partial Nephrectomy
}

Jim Shen1, David Ruckle1, Roger Li1, Mohamed Keheila1, Muhannad Alsyouf1, Jared Schober1, David Tryon1, Phillip Stokes', Herbert C. Ruckle1, Pedro Baron2, Michael De Vera22, D. Duane Baldwin1

${ }^{1}$ Loma Linda University Faculty of Medicine, Department of Urology, Loma Linda, California

2Loma Linda University Faculty of Medicine, Department of Transplantation, Loma Linda, California

J Endourol 2019 Apr 8. doi: 10.1089/end.2018.0654.

\section{EDITORIAL COMMENT}

The amount of renal parenchyma removed during partial nephrectomy plays a major role in the development of surgically induced chronic kidney disease (CKD-S) besides warm ischemia time (WIT). In this retrospective study, in order to document the primary effect of parenchymal volume loss on postoperative renal functions, the authors have compared percent changes in eGFR and incidences of CKD-S in short, intermediate and in the long-term postoperative periods between 250 living kidney donor (DN) and 118 partial nephrectomies (PN) who were followed up to 20.3 months. At baseline, DN patients were younger, mostly female, had lower body mass index, lower American Society of Anesthesiologists physical status classification score and higher preoperative eGFR (all $p<0.001$ ). At hospital discharge, intermediate follow-up, and latest follow-up, DN group had suffered a more intense decline in postoperative renal function at all three time points. Interestingly over 40\% of the DN patients had developed stage 3 or higher CKD-S postoperatively, significantly higher than in the PN group although having much better preoperative eGFRs. Furthermore on subgroup analysis, both subgroups with WIT 1-30 minutes and 31-60 minutes had less renal function loss at all time points compared to DN group ( $<<0.001$ ). This study emphasizes the importance of preservation of the renal parenchyma as much as possible and makes it the number one priority when taken into consideration with other factors including age, body mass index, comorbidities, preoperative renal function and WIT. However we also need more studies in order to enlighten the faith of the CKD-S, especially in the live kidney DN population.

Yarkın Kamil YAKUPOĞLU, MD

๑Copyright 2019 by the Association of Urological Surgery / Journal of Urological Surgery published by Galenos Publishing House. 